\title{
Bibliotheken: Orte der kulturellen Vielfalt und Toleranz
}

\section{Der Deutsche Bibliotheksverband setzt Kommissionsarbeit mit neuem Schwerpunkt auf „Bibliotheken und Diversität“ fort}

https://doi.org/10.1515/bd-2021-0067

Anlässlich des bundesweiten „Deutschen Diversity-Tags“ am 18.05.2021 weist der Deutsche Bibliotheksverband e.V. (dbv) auf die notwendige diversitätsorientierte Öffnung von Bibliotheken in den Bereichen Personal, Programm und Publikum hin. Ab Juli 2021 wird der dbv daher die erfolgreiche Arbeit seiner Kommission „Interkulturelle Bibliotheksarbeit“ mit dem neuen Schwerpunkt „Bibliotheken und Diversität“ fortführen. Die Mitglieder dieser Kommission werden Bibliotheken bundesweit dabei unterstützen, das Thema Vielfalt beim Aufbau ihrer Bestände, in ihrem Personal sowie in ihrer Veranstaltungsarbeit noch stärker zu fördern und umzusetzen. Damit setzt der dbv ein Zeichen für gesellschaftliche Vielfalt und Toleranz in Bibliotheken.

Bibliotheken sind ein wichtiger Motor für Chancengerechtigkeit in einer vielfältigen Gesellschaft. Keine andere Einrichtung erreicht mit ihren niederschwelligen Angeboten so viele unterschiedliche Zielgruppen wie Bibliotheken.

Dazu Prof. Dr. Andreas Degkwitz, Bundesvorsitzender des dbv: „Bibliotheken sind zentrale Orte, um die Potentiale unserer pluralistischen Gesellschaft aufzunehmen, Vielfalt zu fördern und dadurch Kommunen und Gemeinden zu stärken. Dafür müssen Bibliotheken nicht nur ihre Bestände, Services und Veranstaltungen vielfältig ausrichten, sondern das Thema Vielfalt auch strukturell und personell stärken. Bibliotheken brauchen dabei die Unterstützung von Politik und Verwaltung, insbesondere bei der Sicherung von finanziellen, personellen und zeitlichen Ressourcen.“

Der dbv unterstützt auch die Empfehlungen der Kulturstiftung des Bundes für eine nachhaltige Diversitätsentwicklung an Kulturinstitutionen „Diversität als Zukunftsfaktor für Kulturinstitutionen“. An dem Programm „360 - Fonds für Kulturen der neuen Stadtgesellschaft“ sind deutschlandweit auch acht Bibliotheken beteiligt. Die Empfehlungen können hier abgerufen werden: https://www. kulturstiftung-des-bundes.de/de/presse/pressemitteilungen/detail/12-05-2021diversitaet_als_zukunftsfaktor_fuer_kulturinstitutionen.html. 


\section{Der Deutsche Bibliotheksverband e.V. (dbv)}

Der Deutsche Bibliotheksverband e.V. (dbv) vertritt mit seinen mehr als 2.100 Mitgliedern bundesweit rund 10.000 Bibliotheken mit 25.000 Beschäftigten und 11 Mio. Nutzer^innen. Sein zentrales Anliegen ist es, Bibliotheken zu stärken, damit sie allen Bürger^innen freien Zugang zu Informationen ermöglichen. Der Verband setzt sich ein für die Entwicklung innovativer Bibliotheksleistungen für Wissenschaft und Gesellschaft. Als politische Interessensvertretung unterstützt der dbv die Bibliotheken insbesondere auf den Feldern Informationskompetenz und Medienbildung, Leseförderung und bei der Ermöglichung kultureller und gesellschaftlicher Teilhabe für alle Bürger`innen.

Mehr Informationen finden Sie unter www.bibliotheksverband.de.

\section{Pressekontakt}

Kristin Bäßler

Leitung Kommunikation / Pressesprecherin

Tel.: + 49 (0)30 6449899 25 | E-Mail: baessler@bibliotheksverband.de 